University of Nebraska - Lincoln

DigitalCommons@University of Nebraska - Lincoln

\title{
Plant sterol and stanol substrate specificity of pancreatic cholesterol esterase
}

\author{
Andrew W. Brown \\ University of Nebraska-Lincoln \\ Jiliang Hang \\ University of Nebraska-Lincoln \\ Patrick H. Dussault \\ University of Nebraska-Lincoln, pdussault1@unl.edu \\ Timothy P. Carr \\ University of Nebraska-Lincoln, tcarr2@unl.edu
}

Follow this and additional works at: https://digitalcommons.unl.edu/chemistrydussault

Part of the Chemistry Commons

Brown, Andrew W.; Hang, Jiliang; Dussault, Patrick H.; and Carr, Timothy P., "Plant sterol and stanol substrate specificity of pancreatic cholesterol esterase" (2010). Patrick Dussault Publications. 16. https://digitalcommons.unl.edu/chemistrydussault/16

This Article is brought to you for free and open access by the Published Research - Department of Chemistry at DigitalCommons@University of Nebraska - Lincoln. It has been accepted for inclusion in Patrick Dussault Publications by an authorized administrator of DigitalCommons@University of Nebraska - Lincoln. 


\title{
Plant sterol and stanol substrate specificity of pancreatic cholesterol esterase
}

\author{
Andrew W. Brown, ${ }^{1}$ Jiliang Hang, ${ }^{2}$ Patrick H. Dussault, ${ }^{2}$ and Timothy P. Carr ${ }^{1}$ \\ 1. Department of Nutrition and Health Sciences, University of Nebraska-Lincoln, Lincoln, NE 68583-0806, USA \\ 2. Department of Chemistry, University of Nebraska-Lincoln, Lincoln 68583-0304, NE, USA
}

Corresponding author - T. P. Carr, tel 402 472-7940, fax 402 472-1587, email tcarr2@unl.edu

\begin{abstract}
Consumption of plant sterols or stanols (collectively referred to as phytosterols) and their esters results in decreased lowdensity lipoprotein cholesterol, which is associated with decreased atherosclerotic risk. The mechanisms by which phytosterols impart their effects, however, are incompletely characterized. The objective of the present study is to determine if pancreatic cholesterol esterase (PCE; EC 3.1.1.13), the enzyme primarily responsible for cholesterol ester hydrolysis in the digestive tract, is capable of hydrolyzing various phytosterol esters and to compare the rates of sterol ester hydrolysis in vitro. We found that PCE hydrolyzes palmitate, oleate and stearate esters of cholesterol, stigmasterol, stigmastanol and sitosterol. Furthermore, we found that the rate of hydrolysis was dependent on both the sterol and the fatty acid moieties in the following order of rates of hydrolysis: cholesterol $>($ sitosterol $=$ stigmastanol $)>$ stigmasterol; oleate $>($ palmitate $=$ stearate). The addition of free phytosterols to the system did not change hydrolytic activity of PCE, while addition of palmitate, oleate or stearate increased activity. Thus, PCE may play an important but discriminatory role in vivo in the liberation of free phytosterols to compete with cholesterol for micellar solubilization and absorption.
\end{abstract}

Keywords: lipase, phytosterols, plant sterols, plant stanols, cholesterol metabolism, cholesterol absorption

\section{Introduction}

Cardiovascular diseases are collectively the leading causes of death in the United States, with diseases of the heart accounting for over $25 \%$ of all deaths in the United States [1]. Elevated circulating low-density lipoprotein (LDL) cholesterol has long been considered a risk factor for the development of atherosclerotic lesions, which may ultimately lead to impaired blood circulation, heart attacks and strokes. Several pharmaceutical and nutraceutical therapies are presently available to decrease LDL cholesterol, including the consumption of plant sterol and stanol esters (here, collectively referred to as phytosterol esters) [2].

Presently, one mechanism by which phytosterols are thought to exert their cholesterol-lowering effects is by decreasing the incorporation of cholesterol into micelles [3], thereby decreasing absorption and increasing excretion of cholesterol [4]. In in vitro models, only free phytosterols are shown to be effective in displacing cholesterol [5], while the physiological effects are demonstrated in vivo by both free and esterified phytosterols. This leads to the hypothesis that phytosterol esters must be hydrolyzed to impart their cholesterol-lowering effects, which is supported by the observation that supplementation of phytosterol esters increases the amounts of free phytosterols and cholesterol in feces [4]. Pancreatic cholesterol esterase (PCE; EC 3.1.1.13) has been sug- gested as the enzyme responsible for the hydrolysis of phytosterol esters. To date, however, no research has been conducted to confirm whether PCE hydrolyzes these esters, much less whether PCE selectively hydrolyzes various sterol esters.

Efficient absorption of dietary cholesterol esters is dependent on hydrolysis by PCE, followed by the subsequent solubilization of free cholesterol by gall bladder secretions to form mixed micelles in the intestinal lumen; free phytosterols are also solubilized in this way [5]. Furthermore, the putative intestinal cholesterol transporter Niemann-Pick C1Like 1 (NPC1L1) transports free sterols but not sterol esters [6]. Thus, it appears that the hydrolysis of cholesterol esters is necessary for the cholesterol from these esters to be efficiently micellarized and absorbed, while hydrolysis of phytosterol esters may be necessary to impart their cholesterollowering properties.

PCE is a broad lipid-ester hydrolase, hydrolyzing other lipid carboxyl esters in addition to cholesterol esters $[7,8]$. The hydrolytic activity is not uniform across substrates, however, as the diacylglycerol lipase activity of PCE is greater than its triacylglycerol lipase activity [7], and the phospholipase A1 activity of PCE is greater than its phospholipase A2 activity [8]. Because of the documented differences in substrate specificities, we hypothesized that PCE hydrolyzes phytosterol esters, and that the rate of hydrolysis depends on both the sterol and fatty acid moieties. 


\section{Methods and Materials}

\subsection{Reagents}

Stigmasterol (95\%) and stearoyl chloride $(90 \%)$ were purchased from TCI America (Portland, OR, USA). Sitosterol (75\%), cholesteryl stearate $(96 \%)$, cholesteryl palmitate $(97 \%)$ and palmitoyl chloride $(98 \%)$ were purchased from ACROS Organics (Geel, Belgium). Oleoyl chloride (85\%), sodium cholate hydrate $(99 \%)$, cholesteryl oleate $(98 \%)$, oleic acid $(99 \%)$, stearic acid $(99 \%)$, Sylon BTZ and palladium on carbon (Pd/C, 10\%) were purchased from Sigma-Aldrich (St. Louis, MO, USA). 5a-Cholestane was obtained from Steraloids (Newport, RI, USA). Cholesterol (95\%) was obtained from Mallinckrodt OR (Paris, KY, USA). W2 Raney Nickel (RaNi) was prepared by reaction of $\mathrm{NiAl}_{2}$ alloy and $\mathrm{NaOH}$ as previously described [9]. Palmitic acid $(99+\%)$, Triton X-100 and porcine pancreatic cholesterol esterase (Cat. no. 0215067180) were obtained from MP Biomedicals (Irvine, CA, USA). PCE contained the trace enzyme contaminants of $0.0098 \%$ chymotryp$\sin ,<0.0004 \%$ glucose oxidase, $0.432 \%$ trypsin and $<0.0001 \%$ uricase, as determined by the manufacturer, none of which should alter lipid metabolism.

\subsection{Phytosterol ester preparation}

Stigmasterol was used as supplied by the manufacturer in subsequent preparations.

Stigmastanol (a.k.a. sitostanol) was prepared from stigmasterol as previously described [10,11]. Briefly, $1.06 \mathrm{~g}(1 \mathrm{mmol})$ of $\mathrm{Pd} / \mathrm{C}$ was added to $400 \mathrm{ml}$ of a $45 \mathrm{mM}$ stirred solution of stigmasterol in 2-propanol. The reaction mixture was stirred under an atmosphere (balloon) of hydrogen gas at $60^{\circ} \mathrm{C}$ overnight, after which the $\mathrm{Pd} / \mathrm{C}$ was removed by filtration through a pad of Celite. The resulting solution was concentrated at reduced pressure to provide a quantitative yield of pure stigmastanol $\left(\mathrm{mp} 139-140^{\circ} \mathrm{C}\right.$; literature $140^{\circ} \mathrm{C}$ [12]). The lack of a residual alkene was verified by ${ }^{1} \mathrm{H}$ NMR.

Sitosterol of relatively high purity $(92 \%)$ was prepared through a threestep procedure [10]. First, $4.4 \mathrm{~g}(10 \mathrm{mmol})$ of stigmasterol were hydrogenated using $3.4 \mathrm{~g}$ RaNi in $350 \mathrm{ml}$ of ethyl acetate under an atmosphere (balloon) of hydrogen gas; the reduction step was monitored by gas chromathography/mass spectrometry, using an AT-5 column (Alltech Associates, Deerfield, IL, USA; $0.32 \mathrm{~mm} \times 30 \mathrm{~m}$ ). Once the hydrogenation had consumed most of the stigmasterol (typically $10 \mathrm{~h}$ ), the RaNi was removed by filtration through a pad of Celite and the solvent was removed at reduced pressure. The crude residue was analyzed by ${ }^{1} \mathrm{H}$ NMR and determined to consist of a 7:80:13 mixture of stigmasterol, sitosterol and stigmastanol, based upon the relative integration of the signals at $0.720,0.702$ and 0.671 ppm, respectively. Second, the mixture of sterols was dissolved in $100 \mathrm{ml}$ of ether and treated dropwise with $20 \mathrm{ml}$ of $0.6 \mathrm{M}$ bromine in ether at room temperature. The reaction flask was then stoppered and stored at $-20^{\circ} \mathrm{C}$ for 3 days. Crystals, which were residual stigmastanol (confirmed by TLC), were removed by filtration and the filtrate was concentrated in vacuum. The residue was purified by flash column chromatography with $10 \%$ ethyl acetate in hexane to furnish sitosterol 5,6-dibromide. Third, the dibromide was refluxed with $100 \mathrm{ml}$ of $340 \mathrm{mM}$ excess zinc in 1:1 ethanol:acetic acid for $3 \mathrm{~h}$. Solvent was removed, and $50 \mathrm{ml}$ of water was added; the suspension was extracted with three washes of $50 \mathrm{ml}$ of dichloromethane. The organic layer was dried over sodium sulfate and solvent was removed under reduced pressure. The residue was recrystallized from hot acetone to furnish sitosterol as a white solid $\left(\mathrm{mp} 137-138^{\circ} \mathrm{C}\right.$; literature: $139^{\circ} \mathrm{C}$ [12]). The sitosterol obtained $(1.2 \mathrm{~g}, 29 \%$ yield) was determined to be $92 \%$ pure based upon ${ }^{1} \mathrm{H}$ NMR with impurities of stigmasterol $(5 \%)$ and stigmastanol $(3 \%)$.

Each phytosterol ester was generated as follows (illustrated for sitosteryl palmitate): $5.4 \mathrm{mmol}$ of palmitoyl chloride were added dropwise to a stirred mixture of $3.6 \mathrm{mmol}$ of sitosterol and $0.95 \mathrm{mmol}$ dimethylaminopyridine in $20 \mathrm{ml}$ of dry pyridine at $50^{\circ} \mathrm{C}$. The temperature was then increased to $70^{\circ} \mathrm{C}$ and stirred overnight. The reaction was cooled and diluted with $100 \mathrm{ml}$ of water. The resulting suspension was acidified to a $\mathrm{pH}$ of 3-4 with $3 \mathrm{M} \mathrm{HCl}$ and subsequently extracted with three washes of $100 \mathrm{ml}$ of dichloromethane. The combined organic layers were dried over sodium sulfate and solvent was removed under reduced pressure. The residue was recrystallized from hot ethyl acetate to furnish sitosteryl palmitate ( $2.2 \mathrm{~g}$, $88 \%$ yield) as a white solid (mp $79-80^{\circ} \mathrm{C}$; literature: $\left.85.5^{\circ} \mathrm{C}[12]\right)$. Analysis by ${ }^{1} \mathrm{H}$ NMR suggested the sitosteryl palmitate was $92 \%$ pure and contained approximately $8 \%$ of a mixture of stigmasteryl and stigmastanyl palmitates.

\subsection{PCE activity assay}

Lyophilized PCE (100 U) was dissolved in $1 \mathrm{ml}$ of $100 \mathrm{mM}$ potassium phosphate buffer at $\mathrm{pH} 7.0$ as recommended by the manufacturer, separated into $200 \mu \mathrm{l}$ aliquots and stored at $-80^{\circ} \mathrm{C}$ until ready to use. Prior to use, an aliquot was thawed on ice and diluted to $2 \mathrm{U} / \mathrm{ml}$ of the same buffer. The stability of thawed PCE was determined by storing thawed PCE at $4^{\circ} \mathrm{C}$ for 0,5 , or 7 days. The hydrolytic activity of the enzyme was tested on aliquots of the same solubilized cholesteryl oleate solution and incubated as described later, with hydrolysis being measured as the appearance of free cholesterol by gas chromathography (GC). No changes in activity were seen after 7 days (data not shown); regardless, freshly thawed enzyme was used when possible.

A routine enzyme assay was developed and validated to test the substrate specificity of PCE. Sterol esters $(8 \mu \mathrm{mol})$ were dissolved in chloroform and added to $16 \times 100-\mathrm{mm}$ glass, screw-top tubes, and solvent was evaporated under $\mathrm{N}_{2}$ at $50^{\circ} \mathrm{C}$. Sodium cholate hydrate $(100 \mathrm{mg})$, Triton X-100 $(1 \mathrm{ml})$ and deionized water $(8 \mathrm{ml})$ were added and tubes were capped with PTFE-lined lids. The solution was heated and stirred with a stir bar to $100^{\circ} \mathrm{C}$ until the solution turned white. Solutions were removed from heat and slowly cooled to $60^{\circ} \mathrm{C}$ with stirring, after which $1 \mathrm{ml}$ of a 1 $\mathrm{M}, \mathrm{pH} 7.0$ potassium phosphate buffer was added. The final composition of the mixture was $10 \mathrm{ml}$ of $800 \mu \mathrm{M}$ sterol ester, $1 \%(\mathrm{w} / \mathrm{v})$ sodium cholate, $100 \mathrm{mM}$ phosphate buffer and $10 \%(\mathrm{v} / \mathrm{v})$ Triton X-100. The optimal $\mathrm{pH}$ of the assay was determined to be 7.0 after testing a $\mathrm{pH}$ range of $6.0-8.0$ (see results). Solutions remained transparent with no sedimentation throughout the incubation periods. Using cholesteryl oleate, this mixture was demonstrated to produce a micelle when separated using high performance gel filtration, as previously described by Cohen and Carey [13] and modified by Jesch and Carr [14] (data not shown).

The effects of hydrolysis products on the hydrolysis of cholesteryl oleate by PCE under different simulated conditions were determined by adding unesterified stearic, palmitic or oleic acids or cholesterol, sitosterol, stigmasterol or stigmastanol to solubilized cholesteryl oleate: $0.8 \mathrm{mM}$ individual fatty acids were incorporated into the test system to simulate the fatty acids present from $100 \%$ hydrolysis of test substrates; $16 \mathrm{mM}$ individual fatty acids were used to test the effects of a larger ratio of free fatty acids to sterol ester, such as that present in duodenal contents in the fed state; $2.4 \mathrm{mM}$ free sterol was used to approximate the 3:1 ratio of the Food and Drug Administration (FDA)'s recommended daily consumption of phytosterols as esters (1.3 g/day phytosterol esters $\approx 2 \mathrm{mmol}$ phytosterols) [15] to average cholesterol consumed $(257 \mathrm{mg} /$ day $\approx 0.66 \mathrm{mmol}$ cholesterol) [16].

Aliquots of each solubilized sterol ester $(0.5 \mathrm{ml})$ were added to separate glass screw top tubes and preheated to $37^{\circ} \mathrm{C}$. Dilute PCE $(20 \mu \mathrm{l} ; 0.04$ $\mathrm{U})$ was added to each tube. Reactions were incubated at $37^{\circ} \mathrm{C}$ on a rocking platform. Hydrolysis, as measured by the appearance of free sterol or stanol by GC, proved to be linear through $16 \mathrm{~min}$ of incubation with $R^{2}$ $>0.99$ for each sterol ester; therefore, subsequent incubation times were 8 min to ensure sampling from the linear range of the reaction. Hydrolysis was stopped and lipids were extracted by Folch lipid extraction [17] by addition of $2 \mathrm{ml}$ of ice cold 2:1 chloroform:methanol $(\mathrm{v} / \mathrm{v})$ containing $50 \mu \mathrm{M}$ $5 a$-cholestane as an internal standard for GC analysis. Stopped reaction mixtures were vortexed for $10 \mathrm{~s}$ and centrifuged at room temperature at $1000 \times g$ for $10 \mathrm{~min}$; the aqueous supernatant was aspirated and discarded. The chloroform in the infranatant was evaporated under nitrogen gas at $50^{\circ} \mathrm{C} ; 1 \mathrm{ml}$ of hexanes was then added to each sample, samples were vortexed and the samples were again centrifuged at $1000 \times g$ for $10 \mathrm{~min}$. The hexanes-soluble supernatant was transferred to a GC vial, while any residual aqueous layer remained in the infranatant. Hexanes were evaporated at $50^{\circ} \mathrm{C}$ under nitrogen gas; trimethylsyl derivatives were prepared by addition of $100 \mu \mathrm{l}$ of Sylon BTZ to each dried sample and subsequently transferred to a $300 \mu \mathrm{l} \mathrm{GC}$ vial insert. GC vials were capped using PTFE septa, and samples were allowed to derivatize for at least $30 \mathrm{~min}$. Samples were analyzed by GC using an AT-5 column (Alltech Associates).

\subsection{Statistics}

Cholesteryl oleate hydrolysis by PCE was used as an external standard for each set of reactions. Relative activities were calculated by dividing the rate of hydrolysis of individual sterol esters by the within-set hydrolysis of cholesteryl oleate. Statistics were computed using the mixed procedure of SAS software (version 9.1; SAS Institute, Cary, NC, USA). A two-way analysis of variance using sterol and fatty acid moieties as fixed effects was computed. Least squares means were calculated, and between-sterol and between-fatty acid comparisons were analyzed, as well as individual interaction terms of sterol esters, using the "/pdiff" option of "lsmeans." Bonferroni corrections were made to account for multiple comparisons.

\section{Results}

\subsection{Method validity}

Cholesteryl oleate was most effectively hydrolyzed at $\mathrm{pH}$ 7.0, with $\mathrm{pH} 6.0,6.5,7.5$ and 8.0 retaining $89 \%, 80 \%, 84 \%$ and $72 \%$ activity, respectively, relative to $\mathrm{pH} 7.0$. Thus, all subsequent experiments were performed at $\mathrm{pH}$ 7.0. In addition, no appreciable ester synthesis was observed when PCE was added to a solution of cholesterol and oleic acid (data not shown). Storage of PCE for up to $7 \mathrm{~d}$ at $4^{\circ} \mathrm{C}$ did not alter its activity when measured at 8 and 16 min incubations (data not shown). These preliminary experiments ensure that the hydro- 


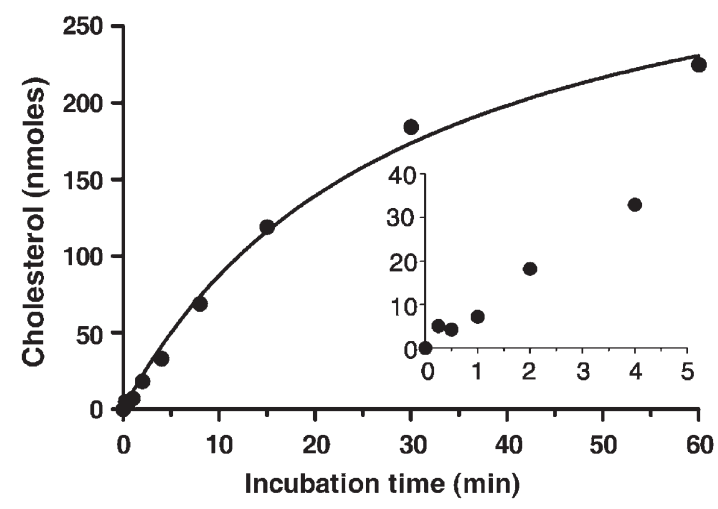

Figure 1. Confirming kinetic properties of PCE in the experimental assay conditions. Cholesteryl oleate was solubilized at a concentration of $800 \mu \mathrm{M}$ in a $100 \mathrm{mM}$, pH 7.0 phosphate buffer containing $1 \%(\mathrm{w} / \mathrm{v})$ sodium-cholate and 10\% (v/v) Triton X-100 and hydrolyzed with PCE at $37^{\circ} \mathrm{C}$ for the times indicated on the $\mathrm{x}$-axis. Progression of hydrolysis was measured by the appearance of free cholesterol. Data points represent one replicate, and are fit with a Michaelis-Menten curve. Inset: Close-up of incubation times less than $5 \mathrm{~min}$.

lytic capabilities of PCE did not change over the course of a set of incubations because freshly thawed PCE was used for incubations whenever possible and was never used when stored at $4^{\circ} \mathrm{C}$ for more than 2 days.

Optimal incubation times were determined by incubating aliquots of a solution of cholesteryl oleate for $0,0.25,0.5,1$, 2, 4, 8, 15, 30 and 60 min (Figure 1). Shorter incubation times, specifically at 0.25 and $0.5 \mathrm{~min}$ (Figure 1 inset), had higher error among replicates and did not fit the overall curve well; longer incubation times, specifically at 30 and $60 \mathrm{~min}$, were beyond the linear region necessary for determining the initial velocity of the reactions. Therefore, subsequent serial reactions for each solubilized sterol ester were conducted with incubation times of $0,1,2,4,8$ and 16 min (Figure 2); hydrolysis was linear for each ester through $16 \mathrm{~min}$. To ensure sampling from the linear range of the reaction, replicate hydrolysis measurements used only the 8-min time point to determine the initial velocity of the reaction.

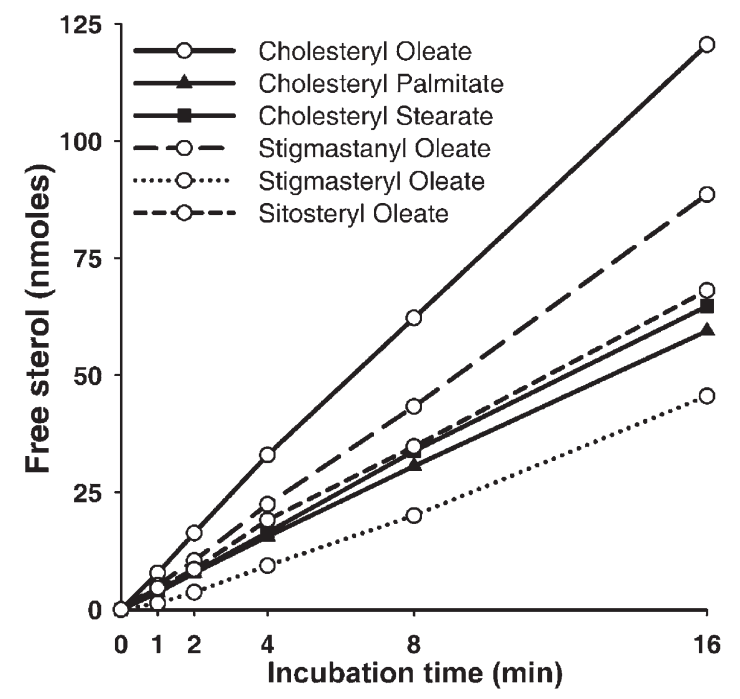

Figure 2. Determining the linear region of hydrolysis by PCE in the experimental assay conditions. Solubilized sterol esters at a concentration of $800 \mu \mathrm{M}$ were hydrolyzed by PCE at $37^{\circ} \mathrm{C}$ for the times denoted on the $x$-axis. Hydrolysis was measured by the appearance of the respective free sterols. For clarity, only cholesterol esters and the oleate esters of the phytosterols are shown, though palmitate and stearate esters of the three phytosterols tested were similarly linear. Data points represent the means of two replicates.

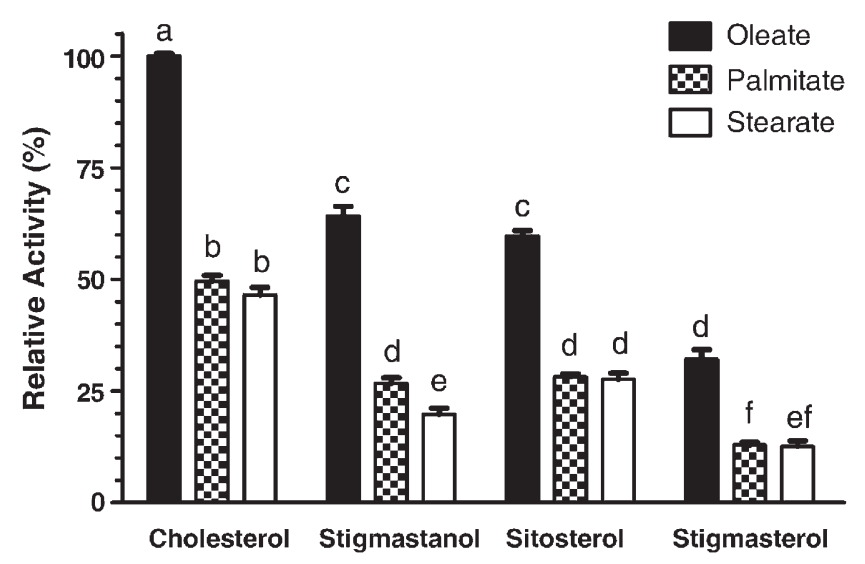

Figure 3. Determining relative rates of hydrolysis of 12 sterol esters. Solubilized sterol esters at a concentration of $800 \mu \mathrm{M}$ were incubated in the presence of PCE at $37^{\circ} \mathrm{C}$ for $8 \mathrm{~min}$. Hydrolysis was measured by the appearance of the respective free sterols. Cholesteryl oleate was used as an external standard for each set of reactions and the average hydrolysis of cholesteryl oleate was set to $100 \%$ for each set of replicates. Bars represent means \pm SEM; $n=4-6$ for all esters except cholesteryl oleate with $n=18$. Bars without common letters differ by a Bonferroni adjusted $P<.05$.

\subsection{Substrate Specificity}

The rate of hydrolysis was affected by both the sterol and the fatty acid portions of the sterol esters. The average rate of hydrolysis of cholesterol esters was significantly greater than the average rate of hydrolysis of the esters of the three phytosterols (Figure 3; sterol effect: $P<.0001)$. Stigmastanol and sitosterol esters were hydrolyzed statistically equally $(56.4 \pm 1.2 \%$ and $58.9 \pm 1.2 \%$, respectively, normalized to cholesterol esters), though more efficiently than stigmasterol esters $(29.3 \pm 1.3 \%)$. Additionally, the rate of hydrolysis of sterol esters was significantly affected by the fatty acid moiety (Figure 3; ester effect: $P<.0001)$. Oleate esters, on average, were hydrolyzed most efficiently, while palmitate and stearate esters were hydrolyzed statistically equally $(45.8 \pm 1.0 \%$ and $41.6 \pm 1.1 \%$, respectively, normalized to oleate esters).

The sterol and ester effects synergistically affected rates of hydrolysis when specific sterol esters were considered (Figure 3;

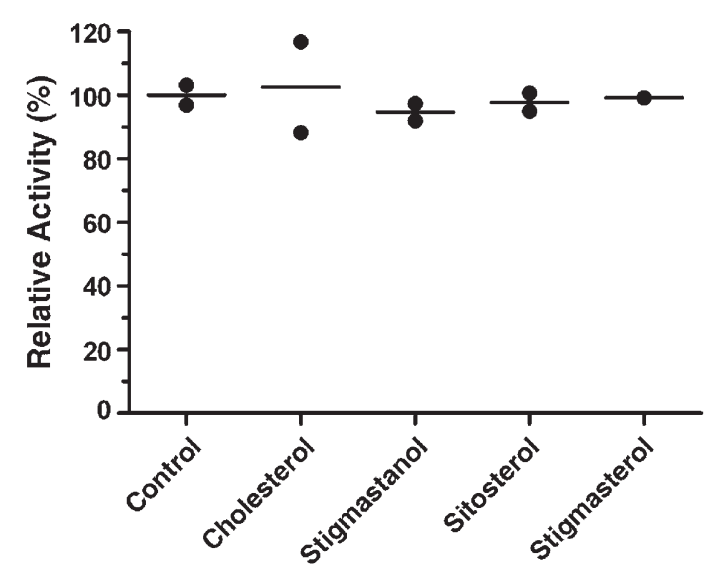

Figure 4. Testing for inhibition of PCE by free sterols. Each sterol was added at a concentration of $2.4 \mathrm{mM}$ to an $800 \mu \mathrm{M}$ solution of cholesteryl oleate and incubated in the presence of PCE for $8 \mathrm{~min}$ at $37^{\circ} \mathrm{C}$. Hydrolysis of cholesteryl oleate was measured by the appearance of free cholesterol. No additional sterol was added to the control, and reactions were normalized to the mean of the control within replicates. No significant differences were observed among the treatments $(P>$ .05). Data points represent single replicates $(n=2)$; horizontal lines represent means. 


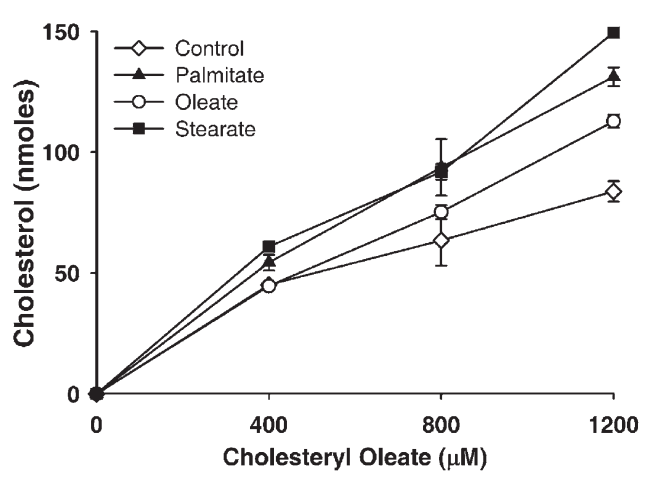

Figure 5. Testing for modulation of PCE activity by free fatty acids. Solubilized cholesteryl oleate at the concentrations denoted on the $x$ axis were hydrolyzed in the presence or absence of $16 \mathrm{mM}$ concentrations of each free fatty acid. Data points represent means of and bars span two replicates.

interaction effect: $P<.0001)$. In all cases, oleate esters were hydrolyzed more efficiently than the palmitate and stearate esters of the same sterol. Further, with the exception of stigmastanyl stearate, hydrolysis of sterol esters was in the order of cholesterol $>$ (stigmastanol $=$ sitosterol $)>$ stigmasterol esters within a particular acyl group; stigmastanyl stearate was hydrolyzed to the same extent as stigmasteryl stearate.

Across all sterol esters tested, cholesteryl oleate was hydrolyzed most effectively, while stigmasteryl palmitate and stearate were hydrolyzed the least effectively $(12.9 \pm 1.3 \%$ and $12.6 \pm 1.6 \%$, respectively, normalized to cholesteryl oleate). Among phytosterol esters, stigmastanyl oleate and sitosteryl oleate were statistically equally hydrolyzed at a greater rate than the other phytosterol esters tested $(64.2 \pm 1.3 \%$ and $59.8 \pm 1.3 \%$, respectively, normalized to cholesteryl oleate).

In an attempt to determine if the differences in rates of hydrolysis were the result of inhibition or activation of PCE by hydrolysis products, free sterols or free fatty acids were added to a solution of cholesteryl oleate. Creating a three-to-one ratio of free sterols to cholesteryl oleate did not affect hydrolysis (Figure 4), nor did the addition of equimolar free fatty acids affect hydrolysis (data not shown). However, hydrolysis appeared to be stimulated when a larger ratio of free fatty acids to sterol esters was used (Figure 5).

\section{Discussion}

Disrupting cholesterol micellarization and absorption in the intestine have been targets for decreasing cholesterol concentrations in the circulation. Ezetimibe, an inhibitor of NPC1L1 [18], and isocoumarin-derived compounds used to inhibit PCE [19] are just two examples of pharmaceutical manipulation of cholesterol absorption with the intent of decreasing atherosclerotic risk. At the forefront of nutraceutical therapies, phytosterols and their esters have been effective in altering intestinal sterol metabolism, though the complete characterization of their mechanisms of action has been elusive. In the present study, we created an in vitro model to investigate the hydrolysis of selected sterol esters by PCE and determined that PCE is capable of hydrolyzing a variety of sterol esters at various rates.

The first consideration in creating our model system was to solubilize sterol esters in an aqueous system devoid of other potential substrates for PCE. PCE is a fairly indiscriminant carboxyl ester hydrolase, capable of hydrolyzing many of the lipids that are used in typical micelle preparations and that exist in micelles in vivo, including phospholipids [7] and acyl glycerols [8]. Furthermore, PCE has been reported to catalyze the reverse reaction of ester synthesis [20], prompting us to create an aqueous model devoid of free fatty acids to avoid encouraging the reverse reaction of ester synthesis by altering the equilibrium.
To avoid using free fatty acids, acyl glycerols or phospholipids as amphipathic detergents, we chose to use Triton X-100 [21].

Other considerations for the model system included satisfying the bile salt dependency of PCE, and, in particular, the preference for a trihydroxy, rather than dihydroxy, bile salt [22], which was satisfied by the addition of sodium cholate; the potential for $\mathrm{pH}$ to affect the hydrolytic capability of PCE [23]; the potential for concurrent production and hydrolysis of sterol esters, which may have been prevented by the addition of greater than $20 \mathrm{mM}$ sodium cholate [24] and the ability to solubilize all esters at equal concentrations. We not only addressed these concerns but validated that PCE can perform enzymatic hydrolysis in our model system in a reproducible manner.

Once the model hydrolysis system was validated, we demonstrated that PCE, in addition to hydrolyzing various sterol esters, exhibited substrate specificity that was affected both by the sterol and fatty acid portions of the ester. Saturated esters were less well hydrolyzed than the unsaturated ester, with the within-sterol palmitate and stearate esters being hydrolyzed approximately $40 \%$ as well as the oleate ester. A similar disparity was demonstrated with a cholesterol esterase derived from rat testis, where cholesteryl stearate was hydrolyzed only $25 \%$ as well as cholesteryl oleate [25]. Substrate specificity of PCE also appeared to be affected by certain structural elements of the individual sterols. The ethyl substitution on carbon 24 of the phytosterols is the consistent structural difference between the phytosterols and cholesterol, and the phytosterol esters were consistently hydrolyzed less efficiently than cholesterol esters. The inclusion of the delta 22 double bond, which is the only structural difference between sitosterol and stigmasterol, coincided with a significant decrease in the relative activity of PCE. However, the presence or absence of the delta five double bond, the only structural difference between sitosterol and stigmastanol, appeared to have little effect on the hydrolytic ability of PCE as evidenced by the similar hydrolysis when sitosterol and stigmastanol esters are compared. Thus, it could be that small changes to the side chain bonded to carbon 17 of these sterols may be more integral in conferring substrate specificity of PCE than the cyclic structure, though this would need to be confirmed by the hydrolysis of other sterol esters.

An unexpected result of this study was the increased hydrolysis of cholesteryl oleate observed upon addition of larger concentrations of free fatty acids. The ratio of free fatty acids to sterol ester in these experiments roughly approximated $50 \%$ hydrolysis of the average consumption of dietary triacylglycerols [16] to the FDA's recommended intake of phytosterol esters [15], thus approximating the initial duodenal contents of these components in the fed state. All three fatty acids increased the hydrolysis of cholesteryl oleate as compared with the solution initially devoid of fatty acids. Although not demonstrated statistically, it appeared that the more hydrophobic fatty acids promoted greater increases in PCE activity. An explanation of the activation of PCE by these fatty acids could be the formation of a more native lipid emulsion with which PCE could interact, such as that which may exist when free fatty acids are present in the duodenum in the fed state. This hypothesis is supported by the observations of Nissinen et al. [26] that only $40 \%$ of plant stanol esters were hydrolyzed in vivo on a low fat diet versus $70 \%$ on a normal fat diet.

Precedence exists for mammalian systems to distinguish between cholesterol and plant sterols, including the higher rate of plant sterol efflux via ABCG5/G8 cotransporters [27] and the higher rate of esterification of cholesterol as compared with sitosterol in the cytosol of proximal rat intestinal cells [28]. Several human studies have also indicated a potential for discrimination among various sterols. In colectomized patients, ingested cholesterol esters were almost completely hydrolyzed $(95 \%)$ by the time they reached the feces, while $90 \%$ of sitosterol esters and only $57 \%$ of stigmastanol es- 
ters were hydrolyzed [26]. In another study, the effects of phytosterol esters on proximal digestion and absorption were isolated using duodenal infusion of solubilized sterols and sterol esters and measuring the composition in the proximal jejunum. The percentage of esterified sitosterol dropped from $64 \%$ to $27 \%$ and esterified stigmastanol from $92 \%$ to $39 \%$ from infusion to the proximal jejunum [29]. While these studies support the present findings, confounding factors such as exposure to multiple digestive enzymes, variable emulsion structures and diverse concentrations of substrates have made it impossible to conclusively determine the hydrolytic activity of PCE alone.

The interaction of phytosterol esters with PCE alone has the potential to decrease the hydrolysis of dietary cholesterol esters via competitive inhibition and thereby decrease the absorption of the free cholesterol derived from cholesterol esters. However, only a small portion of dietary cholesterol is in the esterified form [14]; furthermore, the effects of phytosterol esters are demonstrated whether consumed only once daily or multiple times daily [30], which indicates that competition for PCE is likely not the only mechanism of action for phytosterols. Because the hydrolysis of cholesterol esters would only minimally add to the total cholesterol in the duodenum, competitive inhibition experiments were not conducted in this study.

Here, we have used an in vitro model to demonstrate the potential for vastly different efficacies of phytosterol ester supplementation on cholesterol absorption that depends on phytosterol ester structure. However, it is unknown if the in vitro hydrolysis of phytosterol esters reflects hydrolysis in vivo, nor is it known by what mechanisms phytosterol ester supplementation most effectively decreases plasma cholesterol. Several proposed mechanisms include phytosterols interacting with cholesterol transporters, competing with cholesterol for micellar solubility, regulating cholesterol-related genes and interacting with digestive enzymes [14]. The evidence supporting these mechanisms often has not explored whether phytosterol esters or free phytosterols are most effective at inhibiting cholesterol absorption. The present study demonstrated that phytosterol esters interact with at least one digestive enzyme, PCE, while free phytosterols show no effect on cholesterol ester hydrolysis, helping to further understand the mechanisms behind the ability of phytosterol and phytosterol ester supplementation to inhibit cholesterol absorption.

Acknowledgments - We would like to thank Christopher Ferrel for his help in processing and analyzing the PCE incubations. A.W.B. and J.H. were supported by USDA-NRI competitive grant no. 2007-35200-18298. The research was also supported in part by the University of Nebraska Agricultural Research Division with funds provided through the Hatch Act. Portions of this work were conducted in facilities remodeled with support from NIH (RR016544-01). NMR spectra were acquired, in part, on spectrometers purchased with NSF support (MRI 0079750 and CHE 0091975).

\section{References}

[1] H. Kung, D. L. Hoyert, J. Xu and S. L. Murphy, Deaths: Final data for 2005. Natl Vital Stat Rep, 56 (2008), pp. 1-120.

[2] F. Begemann, G. Bandomer and H. J. Herget, The influence of betasitosterol on biliary cholesterol saturation and bile acid kinetics in man. Scand J Gastroenterol, 13 (1978), pp. 57-63.

[3] E. D. Jesch and T. P. Carr, Sitosterol reduces micellar cholesterol solubility in model bile. Nutr Res, 26 (2006), pp. 579-584.

[4] T. A. Miettinen, M. Vuoristo, M. Nissinen, H. J. Järvinen and H. Gylling, Serum, biliary, and fecal cholesterol and plant sterols in colectomized patients before and during consumption of stanol ester margarine. Am J Clin Nutr, 71 (2000), pp. 1095-1102.

[5] M. J. Armstrong and M. C. Carey, Thermodynamic and molecular determinants of sterol solubilities in bile salt micelles. J Lipid Res, 28 (1987), pp. 1144-1155.

[6] J. M. Brown, L. L. Rudel and L. Yu, Npc111 (niemann-pick c1-like 1) mediates sterol-specific unidirectional transport of non-esterified cholesterol in mcardle-rh7777 hepatoma cells. Biochem J, 406
(2007), pp. 273-283.

[7] O. Zschörnig, M. Pietsch, R. Süss, J. Schiller and M. Gütschow, Cholesterol esterase action on human high density lipoproteins and inhibition studies: detection by MALDI-TOF MS. J Lipid Res, 46 (2005), pp. 803-811.

[8] Q. Chen, B. Sternby and A. Nilsson, Hydrolysis of triacylglycerol arachidonic and linoleic acid ester bonds by human pancreatic lipase and carboxyl ester lipase. Biochim Biophys Acta, 1004 (1989), pp. 372-385.

[9] R Mozingo, Catalyst, raney nickel, W-2, . Org Synth, 21 (1941), pp. 15-17 Org. Synth. Coll. Vol. 1955;3:181.

[10] H. W. Kircher and F. U. Rosenstein, Hydrogenation of stigmasterol. Lipids, 83 (1973), pp. 101-106.

[11] M. Xie, A. Qiu and Y. He, Catalytic hydrogenation of stigmasterol to sitostanol. Jingxi Huagong, 2110 (2004), pp. 735-737.

[12] A. Kukis and J. M. R. Beveridge, Preparation and physical properties of some plant steryl esters. J Org Chem, 25 (1960), pp. 1209-1219.

[13] D. E. Cohen and M. C. Carey, Rapid (1 hour) high performance gel filtration chromatography resolves coexisting simple micelles, mixed micelles, and vesicles in bile. J Lipid Res, 31 (1990), pp. 2103-2112.

[14] T. P. Carr and E. D. Jesch, Food components that reduce cholesterol absorption. Adv Food Nutr Res, 51 (2006), pp. 165-204.

[15] Health claims: plant sterol/stanol esters and risk of coronary heart disease (CHD), 21 C. F. R. Sect. 101.83 (Apr. 1, 2008).

[16] IOM. Dietary Reference Intakes for Energy, Carbohydrate, Fiber, Fat, Fatty Acids, Cholesterol, Protein, and Amino Acids. Washington DC: National Academies Press; 2005. pp. 1038, 1058.

[17] J. Folch, M. Lees and G. H. Sloane Stanley, A simple method for the isolation and purification of total lipides from animal tissues. $J$ Biol Chem, 226 (1957), pp. 497-509.

[18] M. Garcia-Calvo, J. Lisnock, H. G. Bull, B. E. Hawes, D. A. Burnett and M. P. Braun, et al. The target of ezetimibe is niemann-pick c1like 1 (npc111). Proc Natl Acad Sci U S A, 102 (2005), pp. 8132-8137.

[19] J. J. Heynekamp, L. A. Hunsaker, T. A. Vander Jagt, R. E. Royer, L. M. Deck and D. L. Vander Jagt, Isocoumarin-based inhibitors of pancreatic cholesterol esterase. Bioorg Med Chem, 16 (2008), pp. 5285-5294.

[20] G. V. Vahouny, S. Weersing and C. R. Treadwell, Micellar-solubilized substrates and cholesterol esterase activity in vitro. Arch Biochem Biophys, 107 (1964), pp. 7-15.

[21] R. C. Johnson and S. N. Shah, Evidence for two cholesterol ester hydrolases in human cerebrospinal fluid. J Neurochem, 37 (1981), pp. 594-596.

[22] P. W. Jacobson, P. W. Wiesenfeld, L. L. Gallo, R. L. Tate and J. C. J. Osborne, Sodium cholate-induced changes in the conformation and activity of rat pancreatic cholesterol esterase. J Biol Chem, 265 (1990), pp. 515-521.

[23] A. Nègre, R. Salvayre, P. Rogalle, Q. Q. Dang and L. DousteBlazy, Acyl-chain specificity and properties of cholesterol esterases from normal and Wolman lymphoid cell lines. Biochim Biophys Acta, 918 (1987), pp. 76-82.

[24] M. Ponz de Leon, F. Carubbi, P. Di Donato and N. Carulli, Cholesterol esterase activity of human intestinal mucosa. Dig Dis Sci, $\mathbf{3 0}$ (1985), pp. 1053-1064.

[25] L. A. Durham and W. M. Grogan, Characterization of multiple forms of cholesteryl ester hydrolase in the rat testis. J Biol Chem, 259 (1984), pp. 7433-7438.

[26] M. J. Nissinen, H. Gylling and T. A. Miettinen, Effects of plant stanol esters supplied in a fat free milieu by pastilles on cholesterol metabolism in colectomized human subjects. Nutr Metab Cardiovasc Dis, 16 (2006), pp. 426-435.

[27] S. Tachibana, M. Hirano, T. Hirata, M. Matsuo, I. Ikeda and K. Ueda, et al. Cholesterol and plant sterol efflux from cultured intestinal epithelial cells is mediated by ATP-binding cassette transporters. Biosci Biotechnol Biochem, 71 (2007), pp. 1886-1895.

[28] I. Ikeda, K. Tanaka, M. Sugano, G. V. Vahouny and L. L. Gallo, Inhibition of cholesterol absorption in rats by plant sterols. J Lipid Res, 29 (1988), pp. 1573-1582.

[29] M. Nissinen, H. Gylling, M. Vuoristo and T. A. Miettinen, Micellar distribution of cholesterol and phytosterols after duodenal plant stanol ester infusion. Am J Physiol Gastrointest Liver Physiol, 282 (2002), pp. G1009-G1015.

[30] J. Plat, E. N. van Onselen, M. M. van Heugten and R. P. Mensink, Effects on serum lipids, lipoproteins and fat soluble antioxidant concentrations of consumption frequency of margarines and shortenings enriched with plant stanol esters. Eur J Clin Nutr, 54 (2000), pp. 671-677. 